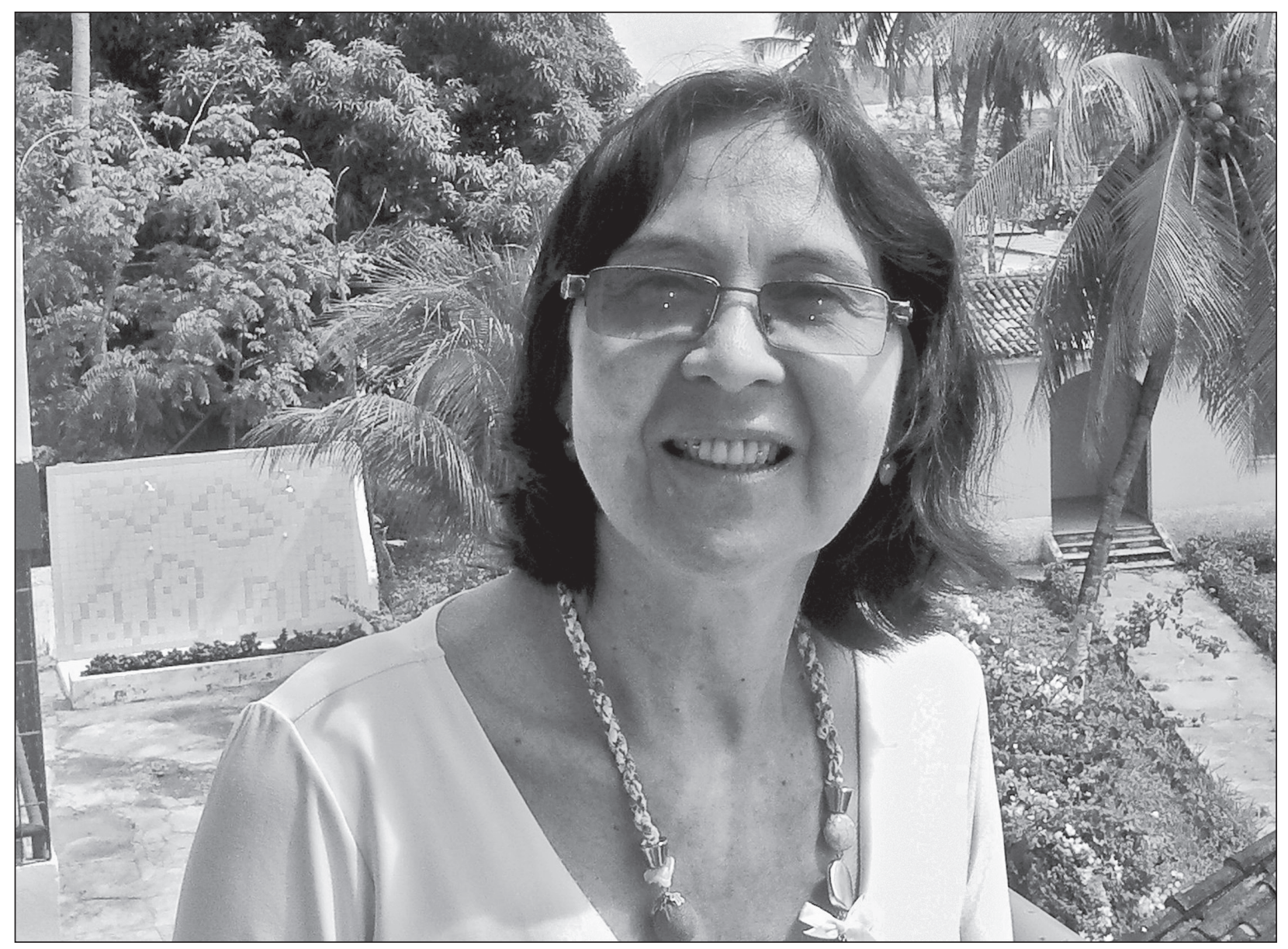

Figure 1. Maria de Jesus da Conceição Ferreira Fonseca (1956-2013). Photo: Luiz Eduardo Ferreira Fonseca. 


\title{
Maria de Jesus da Conceição Ferreira Fonseca (1956-2013): colleague and friend
}

\author{
Ana Yoshi Harada', Luiza Nakayamall \\ 'Museu Paraense Emílio Goeldi/MCTI. Belém, Pará, Brasil \\ "Universidade Federal do Pará. Belém, Pará, Brasil
}

The biologist, Maria de Jesus da Conceição Ferreira Fonseca, known as Jesus (Figure 1). Her large professional life started in the 1980s as a zoologist (Entomologist) student at Museu Paraense Emílio Goeldi (MPEG). However, her polyvalent vision and intention to embrace the world with her hand made her interested in Elementary Education, which she helped to flourish in Belém, where she worked as a fundamental teacher at Secretaria de Estado de Educação (SEDUC) from 1977 to 2007. She did her Specialization degree in Regional education problems (1985), Master's degree in Education (1996), and doctoral degree in Biological Sciences (2003) at the Universidade Federal do Pará (UFPA).

Known as the Pará realist, Jesus worked in multidisciplinary areas, acting in education, research and extension. Thus, she attended courses to work with children and teenagers and develop latu and sensu strictu graduate programs such as "Criação de Mestrado nas Instituições de Ensino Superior - IES" (Rearing Master degree on Higher Education Institutions - HEI). Thereafter, she led the coordination of some actions such as "Projovem Urbano" and "Formação Inicial e Continuada de Formadores" in basic education level, and further collaboration in the creation and coordination of the Biological Diversity in Amazonia course in Pro-Science Program in graduate level, in the Education program (Master's degree) at Universidade do Estado do Pará (UEPA).
As administrator at UEPA, she was the Coordinator for the Centro de Ciências Sociais e Educação (Center for Social Sciences and Education), Master's Degree Program in Education, and Nucleus of Studies in Scientific, Environmental and Social Practices (NECAPS), among others.

In human resources formation at UEPA, she was the advisor for 29 students in start sciences; 29 undergraduate Monographs, 34 Specialization (Short graduate course) and six Master's Degree students. She left the following publications to the Academy: 15 papers in indexed periodics, six organized books, 20 book chapters, ten texts in divulgate Journals and reviews, and 42 abstracts in Scientific Events. She coordinated and collaborated in seven researcher lines, most of them in Education, giving emphasis to "Produção, apropriação e difusão do conhecimento/formação continuada/informática educativa" (Production, approval and knowledge diffusion/ continuous development/educational computing), "Sociobiodiversidade e educação" (Sociobiodiversity and education) and "Saberes culturais e educação na Amazônia" (Cultural know how and education in Amazonia).

She was known for her capacity to help everyone, her collaboration in many committees (at undergraduate and graduate programs and public concourses at research and education institutions), the comments she gave at local, regional and national levels, such as MPEG,

HARADA, A. Y. \& L. NAKAYAMA, 2014. Maria de Jesus da Conceição Ferreira Fonseca (1956-2013): colleague and friend. Boletim do Museu Paraense Emílio Goeldi. Ciências Naturais 9(3): 695-698.

Autor para correspondência: Ana Yoshi Harada. Museu Paraense Emilio Goeldi/MCTI. Programa de Pós-Graduação em Zoologia. Av. Perimetral, 1901 - Terra Firme. Belém, PA, Brasil. CEP 66077-530 (ahara@museu-goeldi.br).

Recebido em 12/11/2014

Aprovado em 01/12/2014

Responsabilidade editorial: Hilton Tulio Costi 
SEDUC, UEPA, UFPA, Secretaria Executiva de Ciência, Tecnologia e Meio Ambiente (SECTAM), her role as an Associação Nacional de Pós-Graduação e Pesquisa em Educação (ANPED) membership, among others. Also, she was a collaborator in politic-pedagogical projects at some public and private institutions, such as prefectures and secretaries. It is relevant to mention her large collaborations at the academy to everyone who sought her help and the advice and support she gave while always smiling and holding confidence to help them.

As a good professional, she received seven prizes and many honors. Thus, the fields of Education and Science lost a researcher and educator that loved her activities and worked with impeccable ethics and respect to all.

Her activities for Environmental Education stimulated everyone, and the proud members of NECAPS, "necapianos", as an exemplary leader and founder. Her way to fight for the ideal "We are the future that we seek", motto, once spoken by Gandhi, was suitably shown on a banner during her last homage.

And we, like friends of everyday, only have to thank for the great personal and professional legacy she allowed us to share.

\section{CHRONOLOGICAL ORDER OF PUBLICATIONS BY MARIA DE JESUS CONCEIÇÃO FERREIRA FONSECA}

FONSECA, M. J. C. F., A. A. S. CORDEIRO, E. A. SILVA \& E. E. F. SILVA, 2012. Ações educativas para a inclusão social da juventude: 1. ed. Gráfica Grafite, Belém.

FONSECA, M. J. C. F. \& S. C. J. SILVA, 2012. Temas amazônicos no contexto escolar: 1. ed.: 1-100. Gráfica Grafite, Belém.

FONSECA, M. J. C. F., V. S. C. NERY, J. T. N. BRITO \& G. C. M. BANDEIRA, 2012. Vivências cotidianas: possibilidade de inserção de temas amazônicos na educação escolar. In: M. J. C. F. FONSECA \& I. C. J. SILVA (Orgs.): Temas amazônicos no contexto escolar: 1. ed.: 52-63. Gráfica Grafite, Belém.

FONSECA, M. J. C. F., 2012. Natureza como mediadora do pensar/ fazer educação em contextos amazônicos. In: M. J. C. F. FONSECA \& S. C. J. SILVA (Orgs.): Temas amazônicos no contexto escolar: 1. ed.: 83-93. Gráfica Grafite, Belém.
FONSECA, M. J. C. F. \& A. A. S. CORDEIRO, 2012. Educação, juventude e inclusão social. In: A. A. S. CORDEIRO, E. A. SILVA, É. E. F. SILVA \& M. J. C. F. FONSECA (Orgs.): Ações educativas para inclusão social da juventude: 1. ed.: 18-27. Gráfica Grafite, Belém.

FONSECA, M. J. C. F., V. S. C. NERY \& G. C. M. BANDEIRA, 2012. Formação inicial de educadores por meio de projetos temáticos. In: A. A. S. CORDEIRO, E. A. SILVA, É. E. F. SILVA \& M. J. C. F. FONSECA (Orgs.): Ações educativas para inclusão social da juventude: 1. ed.: 60-70. Gráfica Grafite, Belém.

FONSECA, M. J. C. F., S. C. J. SILVA, N. N. HORA, C. S. CARVALHO, M. C. DIAS \& E. A. SILVA, 2012. Saberes necessários para inclusão de temas amazônicos no contexto escolar. In: M. J. C. F. FONSECA \& S. C. J. SILVA (Orgs.): Temas amazônicos no contexto escolar: 1. ed.: 9-36. Gráfica Grafitte, Belém.

OLIVEIRA, I. A., M. S. A. F. FRANÇA, T. R. L. SANTOS, M. J. C. F. FONSECA, J. C. MOTANETO, M. J. L. SILVA, M. G. SILVA, M. S. P. LIMA \& A. P. C. SANTOS, 2011. Educação em classes multisseriadas na Amazônia: singularidade, diversidade e heterogeneidade: 1. ed.: 1-168. EDUEPA, Belém.

FRANÇA, M. S. A. F., I. A. OLIVEIRA, T. R. L. SANTOS, M. J. C. F. FONSECA, J. C. MOTA NETO \& M. G. SILVA, A. P. C. SANTOS, M. J. L. SILVA \& M. S. P. LIMA, 2011. Educação em classes multisseriadas na Amazônia: singularidade, diversidade e heterogeneidade: 1. ed.: 1-167. EDUEPA, Belém.

JÚNIOR, J. R. C., M.J. C. F.FONSECA, L. NAKAYAMA\&A. R. SANTANA, 2011. O conhecimento etnoecológico dos pescadores Yudjá, Terra Indígena Paquiçamba, volta grande do Rio Xingu. Tellus (2)1: 123-147.

FONSECA, M. J. C. F. \& I. A. OLIVEIRA, 2011. Cinco anos do PPGED-UEPA na Amazônia: trajetórias, avanços e possibilidades acadêmicas. Revista Cocar 9: 7-16.

FONSECA, M. J. C. F. \& I. A. OLIVEIRA, 2011. A lógica antropocêntrica venceu. Jornal o Sopro (10 out. 2011) 6: 3.

FONSECA, M. J. C. F. \& L. NAKAYAMA, 2010. Narrativas para ensinar-aprender a Amazônia: uma contribuição à educação ambiental em contextos educacionais diversos. Revista de Estudos Universitários 36(3): 143-153.

FONSECA, M. J. C. F., I. A. OLIVEIRA \& T. R. L. SANTOS, 2010. A entrevista na pesquisa educacional. In: I. A. OLIVEIRA, M. I. MARCONDES \& E. TEIXEIRA (Orgs.). Metodologias e técnicas de pesquisa em educação: 1. ed.: 37-53. EDUEPA, Belém.

FONSECA, M. J. C. F., 2009. Rede de saberes em Educação Ambiental: a experiência do Núcleo de Estudos em Educação Científica, Ambiental e Práticas Sociais - NECAPS. Meio Ambiente e Saúde (Faculdade do Futuro) 14: 93-99.

SILVA JÚNIOR, A. S., G. C. M. BANDEIRA \& M. J. C. F. FONSECA (Orgs.), 2009. Vivências pedagógicas em educação científica, ambiental e saúde: 1. ed. Gráfica Smith, Belém. 
FONSECA, M. J. C. F., 2009. A proposta educativa do Núcleo de Estudos em Educação Científica, Ambiental e Práticas Sociais. In: C. A. S. SILVA JÚNIOR, G. C. M. BANDEIRA \& M. J. C. F. FONSECA (Orgs.): Vivências pedagógicas em educação científica, ambiental e saúde: 1. ed.: 23-27. Gráfica Smith, Belém.

FONSECA, M. J. C. F., T. S. OLIVEIRA \& S. S. M. SANTOS, 2009. Mural "olho de boto": uma proposta de educomunicação socioambiental no ensino superior. In: C. A. S. SILVA JÚNIOR, G. C. M. BANDEIRA \& M. J. C. F. FONSECA (Orgs.): Vivências pedagógicas em educação científica, ambiental e saúde: 1. ed.: 50-51. Gráfica Smith, Belém.

FONSECA, M. J. C. F. \& G. M. MONTENEGRO, 2009. Educação ambiental por meio da capoeira: uma ação com jovens da Vila da Barca, Belém, PA. In: C. A. S. SILVA JÚNIOR, G. C. M. BANDEIRA \& M. J. C. F. FONSECA (Orgs.): Vivências pedagógicas sobre educação científica, ambiental e saúde: 1. ed.: 65-67. Gráfica Smith, Belém.

FONSECA, M. J. C. F. \& C. A. S. SILVA JÚNIOR, 2009. A experiência de atividades acadêmicas vivenciadas em grupo/núcleo de pesquisa na formação de jovens pesquisadores. In: C. A. S. SILVA JÚNIOR, G. C. M. BANDEIRA \& M. J. C. F. FONSECA (Orgs.): Vivências pedagógicas em educação científica, ambiental e saúde: 1. ed.: 82-84. Gráfica Smith, Belém.

FONSECA, M. J. C. F., C. A. S. SILVA JÚNIOR, M. C. D. SOUZA \&J. A. SILVA, 2009. Formação de educadores ambientais: uma ação compartilhada na educação superior. In: M. J. C. F. FONSECA, G. C. M. BANDEIRA \& C. A. S. SILVA JÚNIOR (Orgs.): Vivências pedagógicas em educação científica, ambiental e saúde: 1. ed.: 86-90. Gráfica Smith, Belém.

FONSECA, M. J. C. F., C. A. S. SILVA JÚNIOR \& G. M. MONTENEGRO, 2009. Sexualidade e corporeidade: experiência da formação continuada com professores da rede pública do município de Bujarú/PA. In: C. A. S. SILVA JúNIOR, G. C. M. BANDEIRA \& M. J. C. F. FONSECA (Orgs.): Vivências pedagógicas em educação científica, ambiental e saúde: 1. ed.: 105-109. Gráfica Smith, Belém.

FONSECA, M. J. C. F., 2009. Ciência e escola: que relação. O Sopro 9 set. 2009.

FONSECA, M. J. C. F., 2009. As ações de ensino, pesquisa e extensão: a gestão compartilhada do NECAPS. O Sopro 9 set. 2009.

FONSECA, M. J. C. F., 2009. Caminhos de uma prática pedagógica em construção. O Sopro 4 abr. 2009.

FONSECA, M. J. C. F., 2008. A linguagem científica na formação de professores. In: M. P. S. C. SILVA (Orgs.): As interfaces dos estudos Linguísticos: 63-75. UNAMA, Belém.

FONSECA, M. J. C. F. \& D. R. T. RAMOS, 2008. Paternidade juvenil: medos, anseios e a repercussão na família e na escola. Multiplicações 3: 26-32.
MONTENEGRO, G. M. \& M. J. C. F. FONSECA, 2008. Educação Ambiental por meio da Capoeira: uma ação com jovens da Vila da Barca, Belém, Pará. Educação Ambiental em Ação 26: 2-4.

FONSECA, M. J. C. F., 2008. Construindo uma agenda de pesquisa em Educação Científica, Ambiental e Saúde: a experiência do Núcleo de Estudos em Educação Científica, Ambiental e Práticas Sociais. Revista Cocar 2: 25-32.

FONSECA, M. J. C. F., 2008. A proposta educativa do Núcleo de Estudos em Educação Científica, Ambiental e Práticas Sociais. O Sopro 18 dez. 2008.

FONSECA, M. J. C. F., 2008. Por que sopro? O Sopro 20 set. 2008.

FONSECA, M. J. C. F., 2007. A biodiversidade e o desenvolvimento sustentável nas escolas de Ensino Médio de Belém (PA) - Brasil. Educação e Pesquisa 33: 63-80.

FONSECA, M. J. C. F. \& C. A. S. SILVA JÚNIOR, 2007. Conversando sobre sexualidade com a juventude: a prática do Necaps como proposta crítico-reflexiva na formação da juventude. Multiplicações 2: 49-55.

FONSECA, M. J. C. F., 2007. Ações de ensino-pesquisa-extensão em Educação Científica, Ambiental e Saúde: a proposta educativa do Necaps-CCSE-UEPA. Multiplicações 2: 90-94.

FONSECA, M. J. C. F., 2007. Biodiversidade geral e amazônica nas propostas do processo seletivo de ingresso para Universidade do Estado do Pará - UEPA. Revista Cocar 1: 93-98.

FONSECA, M. J. C. F., 2007. Institucionalização do NECAPS: a vitória da persistência. O Sopro 2 fev. 2007.

FONSECA, M. J. C. F., 2006. A leitura da linguagem científica. In: M. P. C. SILVA (Orgs.): As interfaces dos estudos linguísticos: 1. ed.: 95-106. Edunama, Belém.

FONSECA, M. J. C. F., 2005. O lixo nosso de cada dia. In: M. J. C. F. FONSECA (Org.): Caderno de Educação Científica e Ambiental: 1. ed.: 9-27. Smith Produções Gráficas Ltda., Belém.

FONSECA, M. J. C. F., 2004. Biodiversidade amazônica no contexto escolar: algumas considerações. 1. ed.: 279-296. EDUEPA, Belém.

FONSECA, M. J. C. F. \& L. A. R. S. CUNHA, 2004. Alternativas metodológicas para facilitar o ensino de ciências. Comunicação Universitária 5: 71-79.

FONSECA, M. J. C. F., 2003. Educação científica e ambiental através de jogos didáticos. In: F. V. C. FIGUEIREDO \& M. P. C. SILVA (Orgs.): O caráter interdisciplinar da pesquisa: 225-325. KSK Gráfica e Editora, Belém.

FONSECA, M. J. C. F., 1997. Ensino de ciências: um caminho para políticas públicas em educação: o caso de Santa Izabel do Pará. Ver a Educação (3): 67-86.

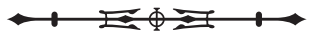


FONSECA, M. J. C. F., 1996. Melhoria da qualidade do ensino de ciências: notas introdutórias. Revista Comunicação Universitária 1(1): 26-29.

FONSECA, M. J. C. F., 1994. Ensino de ciências \& prática pedagógica. Boletim do Núcleo Pedagógico de Apoio ao Desenvolvimento Científico 1: 1-2.
FONSECA, M. J. C. F., 1991. Tele-vício. Jornal do Centro Comunitário Tiradentes 15 ago. 1991.

FONSECA, M. J. C. F., 1991. Estar junto: desafio do trabalho comunitário. Jornal do Centro Comunitário Tiradentes 19 jan. 1991. 